\title{
CATACLYSMIC VARIABLES FROM SLOAN DIGITAL SKY SURVEY. V. THE FIFTH YEAR (2004) ${ }^{1}$
}

\author{
Paula Szkody, ${ }^{2}$ Arne Henden, ${ }^{3,4,5}$ Marcel Agüeros, ${ }^{2}$ Scott F. Anderson, ${ }^{2}$ John J. Bochanski, ${ }^{2}$ Gillian R. Knapp, ${ }^{6}$ \\ Lee Mannikko, ${ }^{2}$ Anjum Mukadam, ${ }^{2}$ Nicole M. Silvestri, ${ }^{2}$ Gary D. Schmidt, ${ }^{7}$ Brian Stephanik, ${ }^{2}$ Todd K. Watson, ${ }^{8}$ \\ Andrew A. West, ${ }^{2}$ Don Winget, ${ }^{9}$ Michael A. Wolfe, ${ }^{2}$ J. C. Barentine, ${ }^{10}$ J. Brinkmann, ${ }^{10}$ Howard J. Brewington, ${ }^{10}$ \\ Ronald A. Downes, ${ }^{11}$ Michael Harvanek, ${ }^{10}$ S. J. Kleinman, ${ }^{10}$ Jurek Krzesinski, $^{10}$ Dan Long, ${ }^{10}$ Eric H. Neilsen, ${ }^{12}$ \\ Atsuko Nitta, ${ }^{10}$ Donald P. Schneider, ${ }^{13}$ Stephanie A. Snedden, ${ }^{10}$ and Wolfgang Voges ${ }^{14}$ \\ Received 2005 October 10; accepted 2005 November 2
}

\begin{abstract}
This paper identifies the cataclysmic variables that appear in spectra obtained in 2004 as part of the Sloan Digital Sky Survey. Spectra of 41 objects, including seven systems that were previously known (CC Cnc, DW Cnc, PQ Gem, AR UMa, AN UMa, RX J1131.3+4322, and UMa 6) and 34 new cataclysmic variables are presented. The positions and ugriz photometry of all 41 systems are given, as well as additional follow-up spectroscopic, photometric, and/or polarimetric observations of eight of the new systems. The new objects include three eclipsing systems, six with prominent He II emission, and six systems that show the underlying white dwarf.
\end{abstract}

Key words: binaries: eclipsing — binaries: spectroscopic — novae, cataclysmic variables — stars: dwarf novae

\section{INTRODUCTION}

At the present time, the Sloan Digital Sky Survey (SDSS; York et al. 2000) has completed Data Release 4, which includes photometry of 180 million objects over $6670 \mathrm{deg}^{2}$ and spectroscopy of over 600,000 objects (Adelman-McCarthy et al. 2006). Information on previous releases is available from Stoughton et al. (2002) and Abazajian et al. (2003, 2004, 2005). ${ }^{15}$ These data sets represent a multitude of objects and scientific programs. While the initial goals of SDSS were related to galaxies and quasars, the large database of stellar spectra and photometry has enabled a vast amount of stellar science as well. Among the latter is the identification of the true population of close binary stars with mass transfer from a late main-sequence star to a white dwarf (cataclysmic variables [CVs]). The SDSS results from the previous releases have revealed a large population of CVs with short orbital periods and mass transfer rates so low that they are sometimes only accreting by winds, and with disks so faint that the underlying stars are revealed (Szkody et al. 2002, 2003a,

\footnotetext{
${ }^{1}$ Based on observations obtained with the Sloan Digital Sky Survey and the Apache Point Observatory $3.5 \mathrm{~m}$ telescope, which are owned and operated by the Astrophysical Research Consortium.

2 Department of Astronomy, University of Washington, Box 351580, Seattle, WA 98195.

US Naval Observatory, Flagstaff Station, P.O. Box 1149, Flagstaff, AZ 86002-1149.

${ }^{4}$ Universities Space Research Association.

5 American Association of Variable Star Observers, 25 Birch Street, Cambridge, MA 02138

6 Princeton University Observatory, Peyton Hall, Princeton, NJ 08544.

7 Steward Observatory, University of Arizona, Tucson, AZ 85721.

${ }^{8}$ Information Technology Services, Southwestern University, Georgetown, TX 78626.

9 Department of Astronomy, University of Texas, Austin, TX 78712.

${ }^{10}$ Apache Point Observatory, P.O. Box 59, Sunspot, NM 88349.

11 Space Telescope Science Institute, 3700 San Martin Drive, Baltimore, MD 21218.

${ }^{12}$ Fermi National Accelerator Laboratory, P.O. Box 500, Batavia, IL 60510.

13 Department of Astronomy and Astrophysics, 525 Davey Laboratory, Pennsylvania State University, University Park, PA 16802.

14 Max-Planck-Institut für extraterrestrische Physik, Geissenbachstrasse 1, D-85741 Garching, Germany.

15 Also see http://www.sdss.org.
}

2003b, 2004a, 2005; Schmidt et al. 2005). A comprehensive review of CVs and all their different subtypes can be found in Warner (1995), while Wickramasinghe \& Ferrario (2000) present a summary of CVs containing magnetic white dwarfs.

The main differences between the SDSS and past surveys lie in its ability to go faint and cover a large amount of sky. While the SDSS is not complete in obtaining spectra of all objects, the large variety of color space sampled by quasars, serendipity, and hot white dwarf categories enables the discovery of a large number of CVs of different types and mass transfer rates. Even though the SDSS spectra themselves provide clues as to the identification of the type of close binary, e.g., strong He I points to a magnetic white dwarf (Polar) or a very high mass transfer rate (SW Sex system), follow-up observations are required to obtain the orbital period and other characteristics that pin down the type of object. The compendium of sources by the end of the survey will provide a good test of stellar evolution models (Howell et al. 2001), and the wide variety of peculiar systems reveals the range of accretion possibilities.

This series of papers is presented to provide an ongoing list of objects for additional study by the astronomical community. In a few cases, we have determined a preliminary period to guide future observations.

\section{OBSERVATIONS AND REDUCTIONS}

Detailed descriptions of the SDSS imaging and spectroscopic instrumentation and reductions can be found in Szkody et al. (2002) and in the papers by Fukugita et al. (1996), Gunn et al. (1998, 2005), Lupton et al. (1999, 2001), Hogg et al. (2001), Smith et al. (2002), Pier et al. (2003), Ivezić et al. (2004), Tucker et al. (2005), and Lupton (2005). Very briefly, SDSS photometric pipelines select objects from colors obtained in five filters $(u, g, r, i$, and $z)$ for spectroscopy covering 3900-9200 $\AA$ at a resolving power of $\sim 1800$. The spectra are wavelength and flux calibrated, and classification (e.g., star, galaxy, quasar) is performed by an automatic software package. We identify CVs using an algorithm that finds objects with Balmer and helium emission/absorption lines, and these objects are then classified by visual inspection. Some plates with visual inspection of all 
TABLE 1

CVs with SDSS SPECTRA

\begin{tabular}{|c|c|c|c|c|c|c|c|c|}
\hline SDSS J ${ }^{\mathrm{a}}$ & MJD-P-F ${ }^{b}$ & $g$ & $u-g$ & $g-r$ & $r-i$ & $i-z$ & $\begin{array}{c}P \\
(\mathrm{hr})\end{array}$ & Comments $^{c}$ \\
\hline $002603.80-093021.0 \ldots$ & $53321-1913-544$ & 20.19 & 0.23 & 0.19 & 0.09 & -0.04 & & \\
\hline $033328.47-000553.5 \ldots \ldots \ldots \ldots \ldots \ldots \ldots \ldots \ldots$ & $53371-2069-158$ & 20.53 & 0.15 & -0.16 & -0.18 & -0.36 & & He II \\
\hline 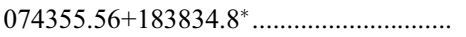 & $52939-1582-302$ & 20.08 & 0.07 & 0.78 & 0.52 & 0.14 & & $\mathrm{H}, \mathrm{L}$ \\
\hline 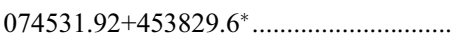 & $53055-1737-253$ & 19.05 & -0.23 & 0.14 & -0.12 & 0.02 & & \\
\hline 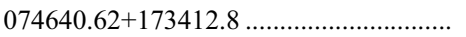 & $53313-1920-305$ & 18.17 & 0.05 & -0.20 & -0.15 & -0.06 & & $\mathrm{H}, \mathrm{L}$ \\
\hline $075117.32+144423.9 \ldots \ldots \ldots \ldots \ldots \ldots \ldots \ldots \ldots \ldots$ & $53350-2080-202$ & 14.18 & -0.06 & 0.18 & 0.05 & -0.03 & 5.19 & PQ Gem IP \\
\hline $075443.01+500729.2 \ldots \ldots$ & $53327-1869-188$ & 17.27 & 0.14 & 0.01 & -0.08 & 0.01 & & He II, NP \\
\hline  & $53350-2080-594$ & 15.33 & -0.53 & 0.40 & -0.08 & 0.03 & 1.435 & DW Cnc IP \\
\hline 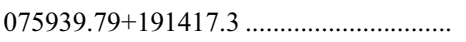 & $53315-1922-172$ & 18.19 & 0.52 & -0.26 & 0.15 & 0.23 & & \\
\hline $080434.20+510349.2^{*} \ldots \ldots \ldots \ldots \ldots$ & $53090-1780-431$ & 17.86 & 0.15 & 0.00 & 0.14 & -0.08 & 1.42 & \\
\hline 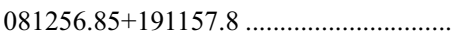 & $53358-2082-344$ & 15.80 & 0.20 & 0.02 & 0.00 & -0.04 & & \\
\hline $082457.15+073702.4^{*} \ldots \ldots \ldots \ldots \ldots \ldots \ldots \ldots \ldots$ & $53084-1758-213$ & 18.57 & 0.56 & -0.13 & -0.15 & -0.06 & & $\mathrm{H}, \mathrm{L}$ \\
\hline 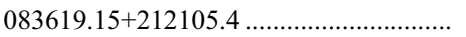 & 53349-1929-001 & 16.77 & 0.41 & 0.21 & 0.09 & 0.05 & 1.76 & $\mathrm{CC} \mathrm{Cnc}$ \\
\hline 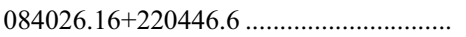 & $53360-2084-172$ & 19.89 & 0.28 & -0.13 & -0.01 & 0.11 & & \\
\hline $084054.15+045519.2^{*} \ldots \ldots$. & $52708-1187-099$ & 15.69 & 0.42 & -0.07 & 0.05 & 0.10 & & \\
\hline 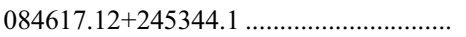 & $53358-1931-487$ & 19.88 & 0.61 & 0.71 & 0.26 & 0.18 & & He II, NP \\
\hline $093214.82+495054.7^{*} \ldots \ldots \ldots \ldots \ldots \ldots \ldots \ldots \ldots$ & $52641-0901-235$ & 17.45 & 0.67 & 0.44 & 0.08 & -0.03 & 10.04 & UMa 6 (NSV18223) \\
\hline $103533.03+055158.4^{*} \ldots \ldots$. & $52636-0999-055$ & 18.82 & 0.32 & 0.03 & -0.18 & -0.14 & & \\
\hline $110014.72+131552.1 \ldots \ldots \ldots \ldots \ldots \ldots \ldots \ldots \ldots \ldots$ & $53358-1750-015$ & 18.67 & -0.36 & -0.11 & 0.24 & 0.16 & & \\
\hline $110425.64+450314.0^{*} \ldots \ldots \ldots \ldots \ldots \ldots \ldots \ldots \ldots$ & $53054-1436-057$ & 15.77 & 0.05 & -0.07 & -0.02 & -0.07 & 1.91 & AN UMa Polar \\
\hline $111544.56+425822.4^{*} \ldots \ldots$. & $53061-1364-115$ & 15.58 & -0.08 & -0.19 & 0.17 & 0.41 & 1.93 & AR UMa Polar \\
\hline $113122.39+432238.6^{* \mathrm{~d}} .$. & $53063-1366-231$ & 16.17 & -0.34 & 0.26 & 0.03 & -0.10 & 1.53 & RX J1131+43 DN \\
\hline $113826.73+061919.5^{*} \ldots \ldots \ldots \ldots \ldots \ldots \ldots \ldots \ldots$ & $53137-1620-290$ & 18.85 & 0.11 & 0.13 & -0.24 & -0.12 & 1.4 & \\
\hline $120231.01+450349.1^{*} \ldots \ldots \ldots \ldots \ldots \ldots \ldots \ldots \ldots$ & $53089-1369-479$ & 19.97 & -0.03 & 0.06 & -0.20 & 0.18 & & \\
\hline $121929.46+471522.8^{*} \ldots \ldots \ldots \ldots \ldots \ldots \ldots \ldots \ldots$ & $53117-1451-176$ & 17.65 & 0.42 & 0.12 & -0.05 & -0.06 & & \\
\hline $122740.83+513925.0^{*} \ldots \ldots \ldots \ldots \ldots \ldots \ldots \ldots \ldots$ & 52374-0884-139 & 19.10 & 0.06 & 0.03 & 0.01 & 0.37 & & \\
\hline 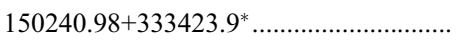 & $53171-1648-408$ & 17.57 & 0.29 & -0.11 & -0.12 & 0.16 & 1.40 & Ec \\
\hline $153213.68+370104.8^{*} \mathrm{e} \ldots \ldots \ldots$. & $53144-1401-443$ & 17.40 & 0.06 & -0.14 & -0.15 & -0.09 & & \\
\hline $155656.92+352336.6^{*} \ldots \ldots \ldots \ldots \ldots \ldots \ldots \ldots \ldots$ & $53139-1417-508$ & 18.39 & 0.16 & 0.09 & -0.07 & -0.10 & 2.14 & Ec \\
\hline $160111.53+091712.7 \ldots \ldots \ldots$ & $53228-1728-551$ & 20.12 & -0.16 & 0.00 & -0.11 & 0.48 & & \\
\hline 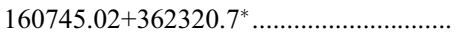 & $53173-1682-582$ & 18.08 & 0.15 & 0.11 & -0.10 & -0.12 & $>3.1$ & Ec, He II \\
\hline $162830.89+240259.1^{*} \ldots \ldots$ & $53177-1572-400$ & 19.79 & 0.14 & 0.08 & -0.04 & 0.20 & & \\
\hline $165359.06+201010.4^{*} \ldots \ldots \ldots \ldots \ldots \ldots \ldots \ldots \ldots$ & $53169-1568-508$ & 17.53 & 0.07 & -0.10 & -0.10 & -0.04 & $P_{\mathrm{sh}}=1.58$ & $\mathrm{DN}$ \\
\hline 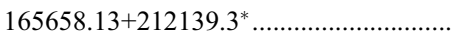 & $52880-1415-095$ & 18.52 & -0.49 & 0.23 & 0.10 & 0.13 & & \\
\hline $165837.70+184727.4^{*} \ldots \ldots \ldots \ldots \ldots \ldots \ldots \ldots \ldots$ & $53172-1567-495$ & 20.08 & 0.42 & -0.04 & 0.03 & 0.42 & & \\
\hline 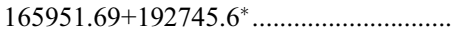 & $53172-1567-578$ & 16.76 & 0.09 & -0.04 & -0.07 & -0.10 & & He II \\
\hline 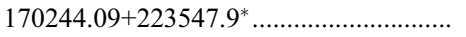 & $53175-1689-342$ & 17.39 & 0.20 & -0.10 & -0.06 & 0.02 & 1.6 & \\
\hline $202520.13+762222.4 \ldots \ldots \ldots \ldots \ldots \ldots \ldots \ldots \ldots \ldots$ & $53240-1661-059$ & 21.83 & 1.23 & 1.32 & 0.52 & 0.43 & & \\
\hline 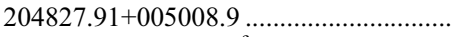 & $53242-1909-601$ & 19.38 & 0.56 & 0.69 & 1.00 & 0.67 & 4.25 & Polar \\
\hline $210449.95+010545.9^{\mathrm{f}} \ldots \ldots \ldots \ldots \ldots \ldots \ldots \ldots \ldots \ldots$ & $53240-1918-347$ & 17.05 & 0.29 & -0.12 & -0.13 & -0.07 & & $\mathrm{H}, \mathrm{L}$ \\
\hline 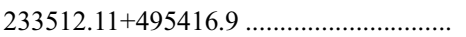 & $53240-1889-524$ & 19.58 & 1.82 & 0.92 & 0.42 & 0.24 & & \\
\hline
\end{tabular}

a Objects marked with an asterisk are publicly available in the SDSS (Adelman-McCarthy et al. 2006).

b MJD-plate-fiber for spectra.

c (DN) dwarf nova; (Ec) eclipsing; (NP) not polarized; (H, L) high and low states.

d Object is northern star of a close pair.

e Object is northwest star of a close pair.

${ }^{\mathrm{f}}$ Object is northeast star of a close pair.

spectra are used to cross-check the algorithm. While our crosschecks show that we recover more than $90 \%$ of the CVs, occasionally misidentification, individual peculiarities, or low signal-to-noise ratio $(\mathrm{S} / \mathrm{N})$ result in a few being missed in the automated search. In this paper we include several systems from previous years (MJDs less than 53,006, i.e., prior to 2004 January 1) that we have recently identified as CVs. Table 1 lists the 34 new CVs along with the seven previously known ones and gives the plate, fiber, and MJD of each spectrum. The coordinates are equinox J2000.0 with the IAU convention of truncation rather than rounding at the given decimal and have an astrometric accuracy of 0.10 . As in the previous papers, the magnitudes and colors are from the point-spread function photometry, with no correction for interstellar reddening. Similar to the past CVs found in SDSS, these are primarily blue objects, with $u-g$ colors near zero, well separated from the main sequence in $u-g$ versus $g-r$ color-color plots (see Szkody et al. [2002, 2003b] for the location of the SDSS stellar locus). To simplify the designations throughout this paper, we refer to the objects as SDSSJ hhmm (hours and minutes of right ascension), except in the case of similar digits requiring differentiation by the addition of the first two digits of declination.

Follow-up observations were obtained for the systems listed in Table 2 (due to exceptionally bad weather, there are less follow-up data than in previous papers). Differential photometry was obtained at the US Naval Observatory Flagstaff Station (NOFS), using a $1024 \times 1024$ SITe/Tektronix CCD on the $1 \mathrm{~m}$ telescope. The observations were made without a filter but 
TABLE 2

FolLOW-UP DATA

\begin{tabular}{|c|c|c|c|c|c|}
\hline SDSSJ & UT Date & Site & Time (UT) & $\begin{array}{l}\text { Exposure } \\
\text { (s) }\end{array}$ & Data Obtained \\
\hline $0754 \ldots$ & 2005 Apr 13 & Bok & $03: 52-04: 25$ & 2200 & Polarimetry $<0.1 \%{ }^{\mathrm{a}}$ \\
\hline $0754 \ldots$ & 2005 Apr 15 & Bok & 04:00-04:21 & 1600 & Polarimetry $<0.4 \%{ }^{\mathrm{a}}$ \\
\hline$\ldots$ & 2005 Jan 14 & MO & $05: 40-010: 41$ & 10 & Photometry \\
\hline 0804 & 2005 May 14 & APO & $02: 40-05: 35$ & 600 & 16 spectra \\
\hline 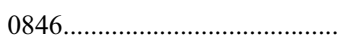 & 2005 Apr 13 & Bok & $04: 37-06: 12$ & 6000 & Polarimetry $<2 \%^{\mathrm{a}}$ \\
\hline 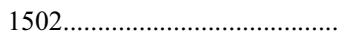 & 2004 Jul 3 & NOFS & 03:36-08:39 & 140 & Photometry \\
\hline $1502 \ldots \ldots \ldots \ldots$ & 2005 May 10 & APO & 08:01-08:51 & 300,600 & Four spectra \\
\hline $1502 \ldots \ldots \ldots \ldots \ldots$ & 2005 Jun 28 & APO & $05: 36-06: 51$ & 600 & Seven spectra \\
\hline $1556 \ldots \ldots \ldots \ldots \ldots \ldots \ldots \ldots$ & $2004 \mathrm{Jul} 7$ & NOFS & $04: 36-09: 24$ & 200 & Photometry \\
\hline $1607 \ldots \ldots \ldots \ldots$ & $2004 \mathrm{Jul} 10$ & NOFS & 03:40-09:17 & 140 & Photometry \\
\hline 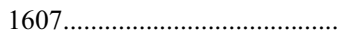 & 2004 Aug 2 & NOFS & $03: 26-07: 43$ & 140 & Photometry \\
\hline 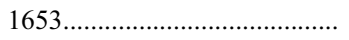 & 2004 Aug 24 & NOFS & 02:45-07:07 & 140 & Photometry \\
\hline 1702 & 2004 Aug 25 & NOFS & 02:43-07:05 & 80 & Photometry \\
\hline
\end{tabular}

${ }^{\text {a }}$ Circular polarization with $3 \sigma$ upper limit.

calibrated with other nights of all-sky photometry using Landolt standards to place comparison stars onto the Johnson $V$ magnitude system.

High-speed time-series photometry on SDSSJ 0804 was acquired using the prime-focus CCD camera Argos (Nather \& Mukadam 2004) on the $2.1 \mathrm{~m}$ telescope at McDonald Observatory (MO) on 2005 January 14. A blue bandpass BG40 filter was used to reduce the contribution of the red sky and improve the $S / N$ for the blue white dwarf. Due to the frame-transfer CCD, back-toback $10 \mathrm{~s}$ exposures with no intervening dead time were obtained.

Time-resolved spectra were obtained for a few objects using the $3.5 \mathrm{~m}$ telescope at the Apache Point Observatory (APO) with the Double Imaging Spectrograph in high-resolution mode (resolution about $2 \AA$ ) with a 1.5 slit. The spectra were reduced to flux and wavelength using standard IRAF ${ }^{16}$ routines. Equivalent widths and fluxes for the Balmer and helium emission lines were measured with the "e" routine in the IRAF SPLOT package, while the velocities were determined with the double-Gaussian method (Shafter 1983), which is best for lines with complicated structure (central absorption or narrow emission components within the broad emission). A sinusoid was then fitted by least squares to the velocities to obtain $\gamma$ (systemic velocity), $K$ (semiamplitude), $P$ (orbital period), and $\phi_{0}$ (phase of crossing from redshift to blueshift).

Circular polarization was measured for two systems, using the CCD spectropolarimeter SPOL with a low-resolution grating on the $2.3 \mathrm{~m}$ Bok telescope on Kitt Peak. The dates and types of follow-up observations are summarized in Table 2.

\section{RESULTS}

Figure 1 shows the SDSS spectra for all 41 systems. Table 3 provides the equivalent widths and fluxes of the prominent hydrogen Balmer and helium emission lines. Following the categories used in the previous papers, we can sort and highlight the systems with identifiable/interesting characteristics.

\subsection{Previously Known Systems}

Among the objects listed in Table 1 are the known CVs: CC Cnc, DW Cnc, PQ Gem, AR UMa, AN UMa, RX J1131.3+

\footnotetext{
${ }^{16}$ IRAF (Image Reduction and Analysis Facility) is distributed by the National Optical Astronomy Observatory, which is operated by the Association of Universities for Research in Astronomy, Inc., under cooperative agreement with the National Science Foundation.
}

4322, and UMa 6 (NSV 18223). ${ }^{17}$ The new low accretion rate Polar that was recently discussed in detail in Schmidt et al. (2005) is also included in this list (SDSSJ 2048) for completeness. AR UMa and AN UMa are known Polars, DW Cnc and PQ Gem are known intermediate Polars (IPs), RX J1131.3+4322 is a known dwarf nova, and UMa 6 is classified as a nova-like star. The SDSS spectra are all similar to past published spectra of these objects, with the exception of UMa 6 (SDSSJ 0932). The spectrum in Munari et al. (1997) shows strong He II in relation to $\mathrm{H} \beta$, but the SDSS spectrum in Figure 1 shows a much larger ratio between these two lines, with $\mathrm{H} \beta$ barely visible. The period quoted in the Downes catalog is very long $(10 \mathrm{hr})$, but the reference is only to an unpublished poster at a $\mathrm{CV}$ conference in Oxford. Thus, there is no way to know if this difference is due to orbital variations or a different state of the system. The $V$ magnitude given in Munari et al. (1997) is 17.1, comparable to the SDSS $g$ mag.

\subsection{High-Inclination Systems}

The indication of high inclination is generally a deep doubling of the Balmer emission lines, especially in the higher order lines. With this criterion, the possible high-inclination systems are SDSSJ 1035, 1227, and 1502 (Fig. 1). Of these three, only SDSSJ 1502 has been followed up with photometry and spectroscopy to confirm that it is a short-period, eclipsing system. SDSSJ 1556 and 1607 also revealed eclipses, although their lines only show some indication of doubling structure in the higher order Balmer lines.

SDSSJ 1502.- -USNO photometry reveals a deep (2.5 mag) eclipse occurring at a period of 84.24 minutes. Figure 2 shows three eclipses on the night of July 3, occurring at HJD 2,453,180+ $9.6921,9.7506$, and 9.8091. The light curve is fairly flat prior to eclipse, indicating the lack of a strong hot spot where the masstransfer stream intersects the disk. The two nights of follow-up spectroscopy were short runs (with the first night halted early due to clouds; Table 2), but an eclipse was covered on each night, and almost the entire orbit was obtained on the second night. The lines show the deep doubling evident in the SDSS spectrum (Fig. 1), and the red and blue components show large variations throughout the orbit, as is typical for high-inclination systems. Because of this

\footnotetext{
${ }^{17}$ References, periods, and characteristics of these systems are available from the online CV catalog of Downes (http://icarus.stsci.edu/ downes/cvcat/).
} 

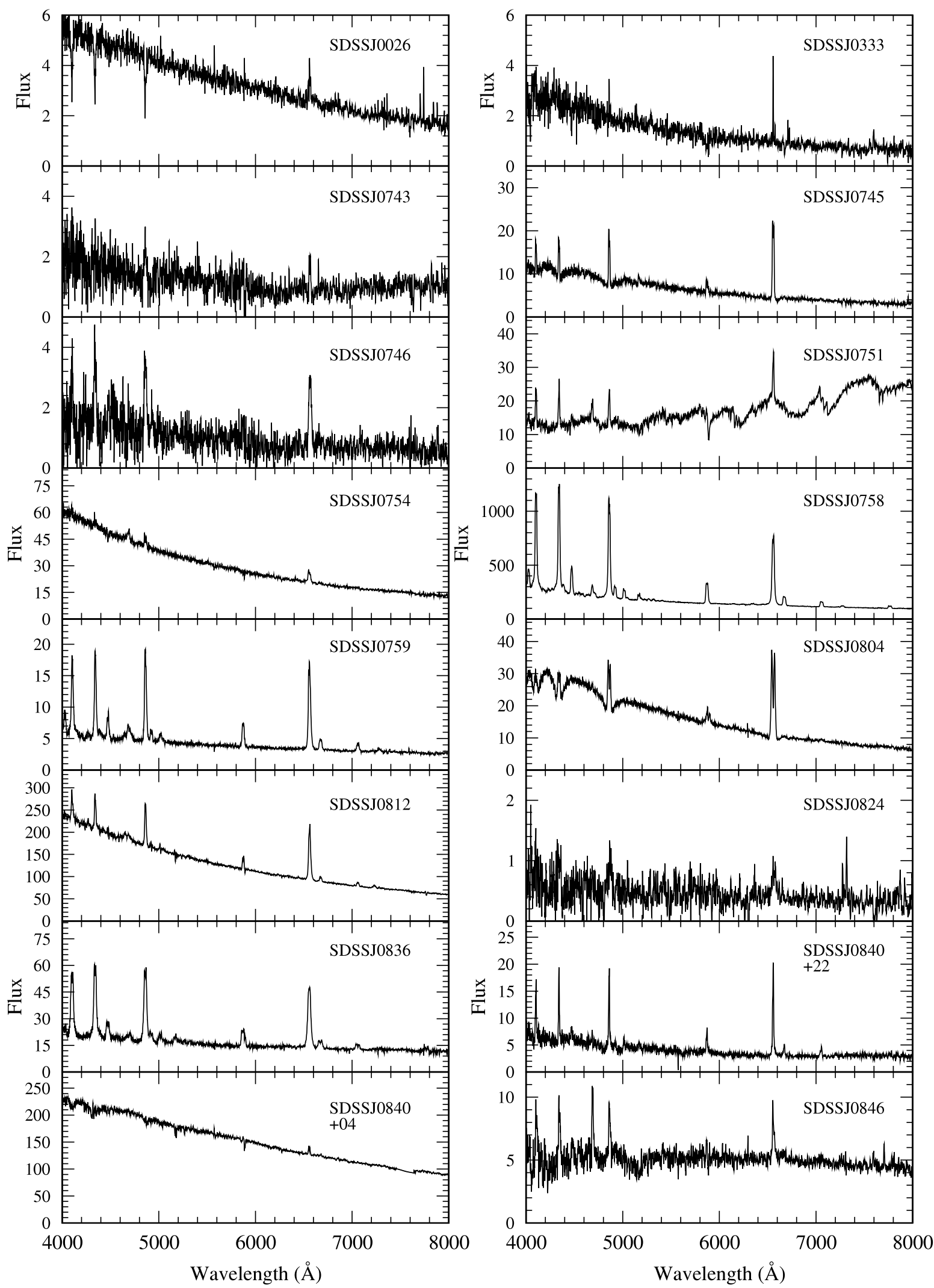

FIg. 1.-SDSS spectra of the $41 \mathrm{CVs}$. The flux scale is in units of flux density $\left(10^{-17} \mathrm{ergs} \mathrm{cm}^{-2} \mathrm{~s}^{-1} \AA^{-1}\right)$. The spectral resolution is about $3 \AA$. 

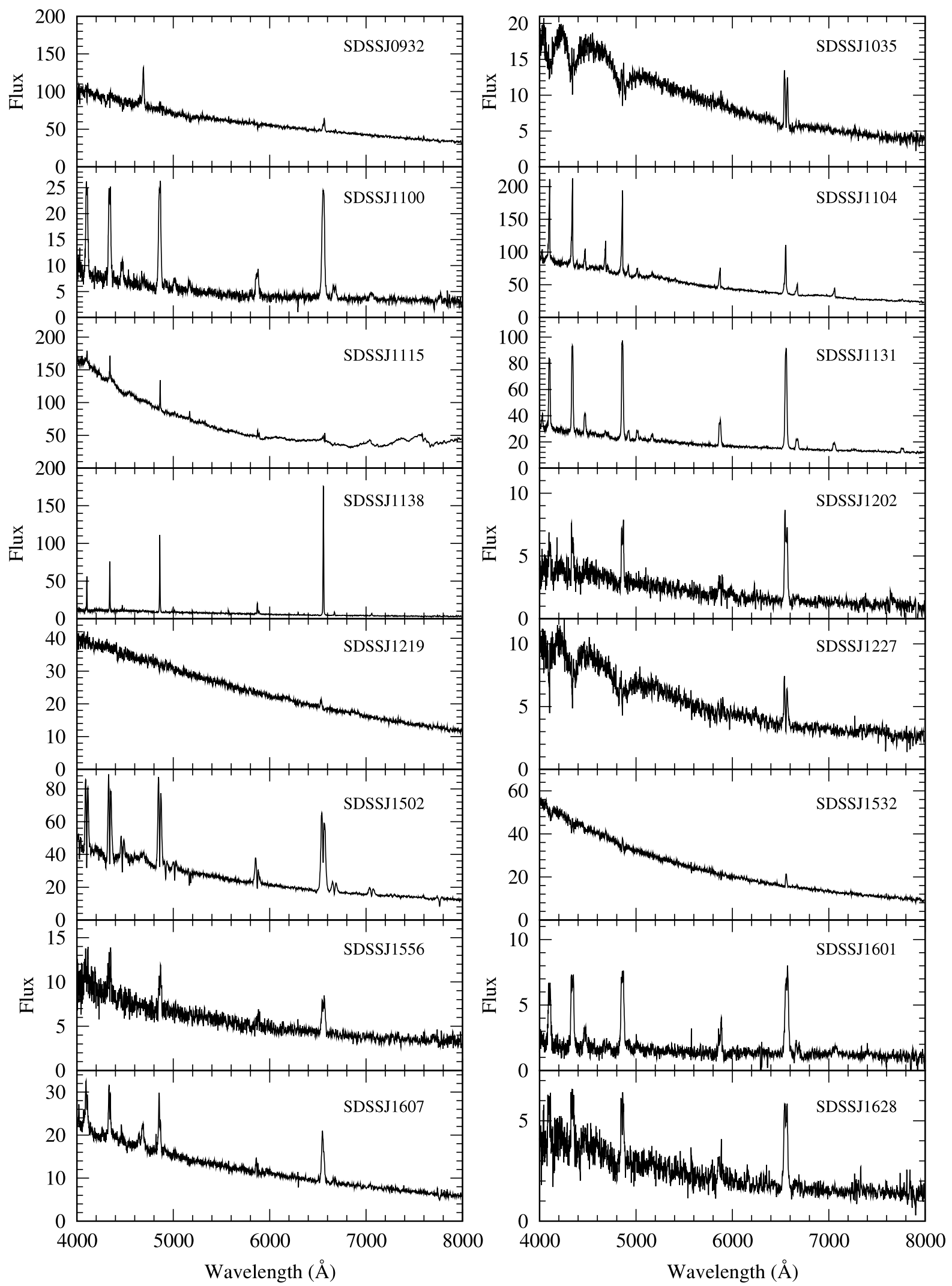

FIg. 1.-Continued 

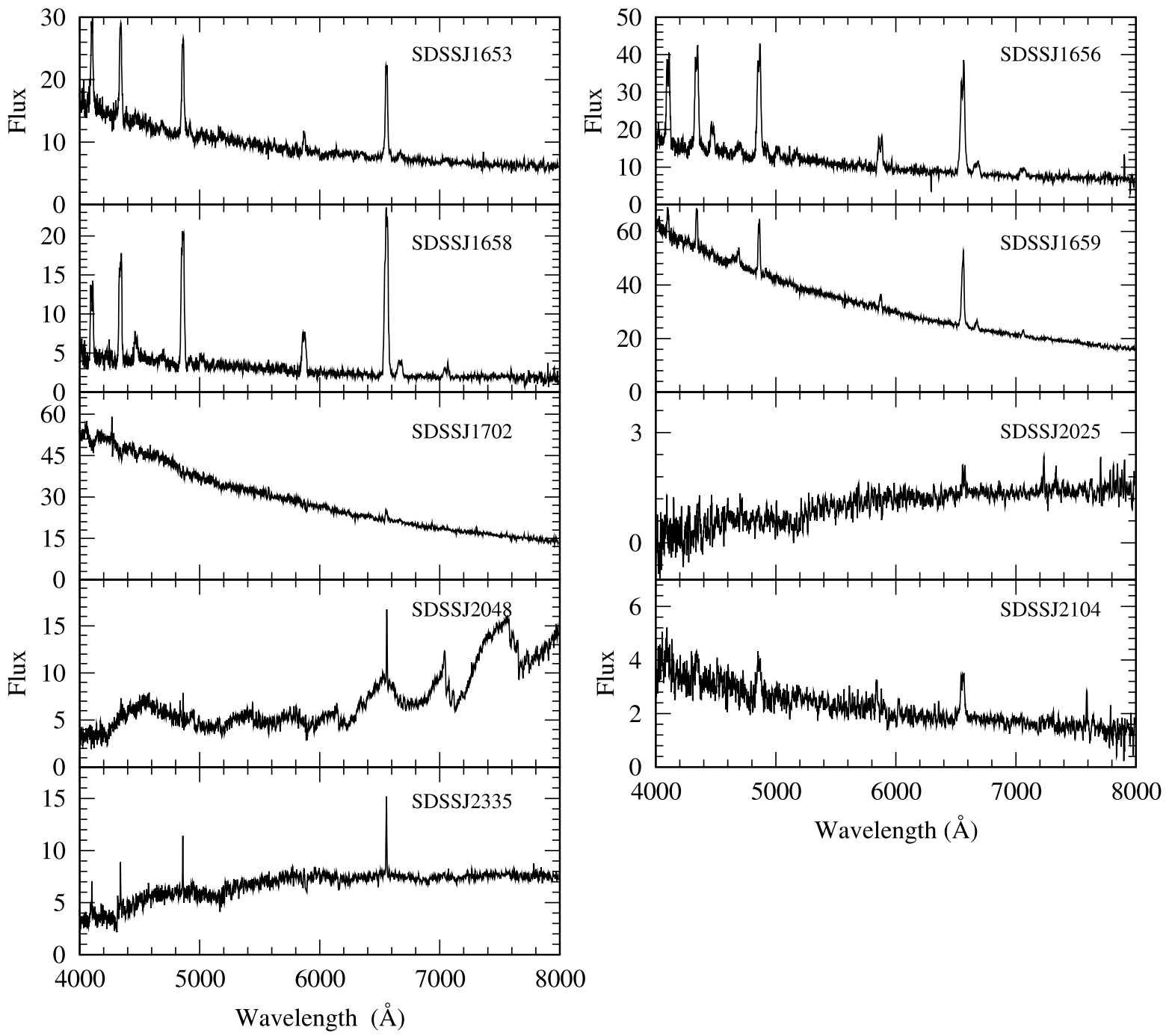

FIG. 1.-Continued

structure, the double-Gaussian method was used to measure the lines, but since the observation length was short, the period was fixed at the photometrically determined value to obtain the best radial velocity solutions shown in Figure 3 and listed in Table 4. Gaussian separations of $2600 \mathrm{~km} \mathrm{~s}^{-1}$ for $\mathrm{H} \beta$ and $2300 \mathrm{~km} \mathrm{~s}^{-1}$ for $\mathrm{H} \alpha$ were used for these solutions.

SDSSJ 1556.-Figure 4 shows the USNO light curve revealing $1.3 \mathrm{mag}$ deep eclipses with a period of $2.14 \mathrm{hr}$ (eclipses occur at HJD 2,453,190+3.7701 and 3.8592). This period is in the gap where few disk CVs exist (Warner 1995), although it is close to the edge. In this case, there is a prominent hump preceding the eclipse, suggesting that a hot spot is present from the stream intersection.

SDSSJ 1607.- In contrast to the above two cases, the $1.5 \mathrm{mag}$ eclipse of SDSSJ 1607 is broad and V-shaped (Fig. 5). There was only one eclipse detected on each of the two nights of observation (at HJD 2,453,196.7444 and 2,453,219.7335). This allows some constraint on the orbital period from the length of the data string on each night. The period must be longer than $3.1 \mathrm{hr}$ (since there was not a repeat eclipse during this stretch on July 10) and likely not much longer than $4 \mathrm{hr}$, as an eclipse was detected on each of the two nights. The shape of the eclipse, the period above the gap, and the spectrum (Fig. 1), which shows a strong blue continuum, strong CN and He II at 4640 and $4686 \AA$, and a strong single-peaked Balmer $\mathrm{H} \alpha$ emission line, are all consistent with this being a SW Sex star. Further confirmation will come if there is absorption present in the Balmer and He I lines at some phases and if there is an offset between the photometric and spectroscopic phases. The structure in the higher order Balmer lines and the absorption in He 4471 present in the 46 minute SDSS exposure (Fig. 1) could be due to the SDSS observation taking place during absorption phases.

\subsection{Dwarf Novae}

Objects that show a large difference in brightness at different epochs of observation could be dwarf novae or nova-like stars undergoing transitions between high and low states of mass transfer. Spectroscopy at the low brightness level compared to the high can determine if the spectra change from strong emission to absorption, hence identifying a dwarf nova. The SDSS photometry, spectroscopy, and follow-up work all provide different epochs of observation, and an additional epoch is obtained from comparison to the Digitized Sky Survey (DSS). There are four cases that show a large difference between the SDSS photometry and spectroscopy (SDSSJ 0743, 0746, 0824, and 2104). In all four, the object is brighter during the photometry than when the spectrum was obtained, and the spectra have the typical appearance of dwarf novae at quiescence (note that the 
TABLE 3

SDSS Emission Line FluXes and Equivalent Widths

\begin{tabular}{|c|c|c|c|c|c|c|c|c|}
\hline \multirow[b]{2}{*}{ SDSSJ } & \multicolumn{2}{|c|}{$\mathrm{H} \beta$} & \multicolumn{2}{|c|}{$\mathrm{H} \alpha$} & \multicolumn{2}{|c|}{ He4471 } & \multicolumn{2}{|c|}{ Не пा4686 } \\
\hline & $F$ & EW & $F$ & EW & $F$ & EW & $F$ & EW \\
\hline $0026 \ldots \ldots \ldots \ldots \ldots \ldots$ & $\ldots$ & $\ldots$ & 0.2 & 8 & $\ldots$ & $\ldots$ & $\ldots$ & $\ldots$ \\
\hline 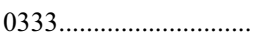 & 0.1 & 5.6 & 0.2 & 18 & $\ldots$ & $\ldots$ & $\ldots$ & $\ldots$ \\
\hline $0743 \ldots \ldots \ldots \ldots \ldots \ldots$ & 0.3 & 28 & 0.2 & 24 & $\ldots$ & $\ldots$ & $\ldots$ & $\ldots$ \\
\hline 0745 & 1.9 & 25 & 3.8 & 91 & $\ldots$ & $\ldots$ & $\ldots$ & $\ldots$ \\
\hline 0746 & 0.7 & 56 & 0.7 & 91 & $\ldots$ & $\ldots$ & $\ldots$ & $\ldots$ \\
\hline 0751......................... & 1.6 & 12 & 1.8 & 9 & 0.3 & 3 & 0.9 & 6 \\
\hline 0754 & 1.4 & 3 & 1.8 & 9 & $\ldots$ & $\ldots$ & 1.1 & 3 \\
\hline $0758 \ldots \ldots$ & 233.9 & 106 & 187.7 & 131 & 57.8 & 25 & 17.7 & 8 \\
\hline 0759 & 3.5 & 72 & 3.8 & 107 & 0.9 & 18 & 0.7 & 14 \\
\hline 0804 & 4.6 & 24 & 10.4 & 106 & $\ldots$ & $\ldots$ & $\ldots$ & $\ldots$ \\
\hline 0812 & 19.1 & 11 & 31.5 & 34 & $\ldots$ & $\ldots$ & 2.9 & 2 \\
\hline $0824 \ldots \ldots \ldots \ldots \ldots \ldots$ & 0.2 & 39 & 0.2 & 45 & $\ldots$ & $\ldots$ & $\ldots$ & $\ldots$ \\
\hline $0836 \ldots \ldots \ldots \ldots \ldots \ldots \ldots \ldots$ & 14.2 & 77 & 13.3 & 92 & 3.1 & 16 & 1.3 & 7 \\
\hline $0840+22 \ldots \ldots \ldots \ldots \ldots \ldots$ & 1.8 & 38 & 2.3 & 73 & 0.4 & 7 & 0.3 & 5 \\
\hline $0840+04 \ldots \ldots \ldots \ldots \ldots \ldots$ & $\ldots$ & $\ldots$ & 2.8 & 2 & $\ldots$ & $\ldots$ & $\ldots$ & $\ldots$ \\
\hline $0846 \ldots \ldots$ & 0.7 & 14 & 0.9 & 17 & 0.4 & 9 & 0.8 & 15 \\
\hline $0932 \ldots \ldots \ldots \ldots \ldots \ldots$ & 1.1 & 2 & 3.0 & 6.3 & $\ldots$ & $\ldots$ & 8.0 & 10 \\
\hline $1035 \ldots \ldots \ldots \ldots \ldots \ldots \ldots$ & 0.5 & 5 & 2.4 & 44 & $\ldots$ & $\ldots$ & $\ldots$ & $\ldots$ \\
\hline 1100 & 5.7 & 98 & 6.7 & 155 & 1.0 & 14.0 & 0.4 & 7 \\
\hline $1104 \ldots \ldots \ldots \ldots \ldots \ldots$ & 14.4 & 21 & 11.5 & 31 & 3.6 & 5 & 4.4 & 6 \\
\hline $1115 \ldots \ldots \ldots \ldots \ldots \ldots \ldots$ & 2.9 & 3 & 0.6 & 1.6 & $\ldots$ & $\ldots$ & & \\
\hline $1131 \ldots \ldots \ldots \ldots \ldots \ldots \ldots \ldots \ldots$. & 15.7 & 69 & 18.1 & 117 & 3.1 & 12 & 0.7 & 3 \\
\hline 1138 & 6.9 & 81 & 13.7 & 228 & 0.4 & 4 & 0.1 & 1 \\
\hline 1202 & 1.1 & 38 & 2.3 & 159 & $\ldots$ & $\ldots$ & $\cdots$ & $\ldots$ \\
\hline $1219 \ldots \ldots \ldots \ldots \ldots \ldots$ & $\ldots$ & $\ldots$ & 0.5 & 2 & $\ldots$ & $\ldots$ & $\ldots$ & $\ldots$ \\
\hline $1227 \ldots \ldots \ldots \ldots \ldots \ldots \ldots$ & 0.5 & 8 & 1.1 & 30 & $\ldots$ & $\ldots$ & $\ldots$ & $\ldots$ \\
\hline $1502 \ldots \ldots \ldots \ldots \ldots \ldots \ldots \ldots \ldots \ldots$ & 16.5 & 49 & 22 & 121 & 2.6 & 7 & 1.1 & 7 \\
\hline $1532 \ldots \ldots \ldots \ldots \ldots \ldots$ & 0.7 & 3 & 0.9 & 6 & .. & $\ldots$ & 0.3 & 0.9 \\
\hline $1556 \ldots \ldots \ldots \ldots \ldots \ldots \ldots \ldots$ & 1.0 & 15 & 2.0 & $35 \ldots$ & & & & \\
\hline 1601_...................... & 2.0 & 105 & 2.5 & 177 & 0.4 & 22.6 & & \\
\hline 1607 & 2.6 & 16 & 3.3 & 36 & 0.3 & 2 & 1.3 & 7 \\
\hline $1628 \ldots \ldots \ldots \ldots \ldots \ldots$ & 1.0 & 33 & 1.9 & 115 & $\ldots$ & $\ldots$ & $\ldots$ & $\ldots$ \\
\hline $1653 \ldots \ldots \ldots \ldots \ldots \ldots \ldots$ & 3.3 & 28 & 3.7 & 49 & $\ldots$ & $\ldots$ & 0.2 & 2 \\
\hline $1656 \ldots \ldots \ldots \ldots \ldots \ldots \ldots \ldots \ldots$ & 9.8 & 73 & 10.8 & 123 & 2.2 & 16 & 1.1 & 8 \\
\hline 1658 & 5.1 & 146 & 7.0 & 309 & 0.8 & 20 & 0.2 & 6 \\
\hline 1659 & 3.7 & 8 & 6.2 & 24 & 0.5 & 1 & 0.9 & 2 \\
\hline $1702 \ldots \ldots \ldots \ldots \ldots \ldots$ & 0.3 & 1 & 0.6 & 3 & $\ldots$ & $\ldots$ & $\ldots$ & $\ldots$ \\
\hline $2025 \ldots \ldots \ldots \ldots \ldots \ldots$ & $\ldots$ & $\cdots$ & 0.1 & 9 & $\ldots$ & $\ldots$ & $\ldots$ & $\ldots$ \\
\hline 2048 & 0.2 & 3 & 0.5 & 6 & $\ldots$ & $\ldots$ & $\ldots$ & $\ldots$ \\
\hline $2104 \ldots \ldots \ldots \ldots \ldots \ldots \ldots \ldots \ldots$ & 0.4 & 16 & 0.6 & 31 & $\ldots$ & $\cdots$ & $\ldots$ & $\ldots$ \\
\hline $2335 \ldots \ldots \ldots \ldots \ldots \ldots \ldots \ldots$ & 0.3 & 4 & 0.5 & 7 & $\ldots$ & $\ldots$ & $\ldots$ & $\ldots$ \\
\hline
\end{tabular}

Note.-Fluxes are in units of $10^{-15} \mathrm{ergs}^{-2} \mathrm{~s}^{-1}$; equivalent widths are in units of $\AA$.

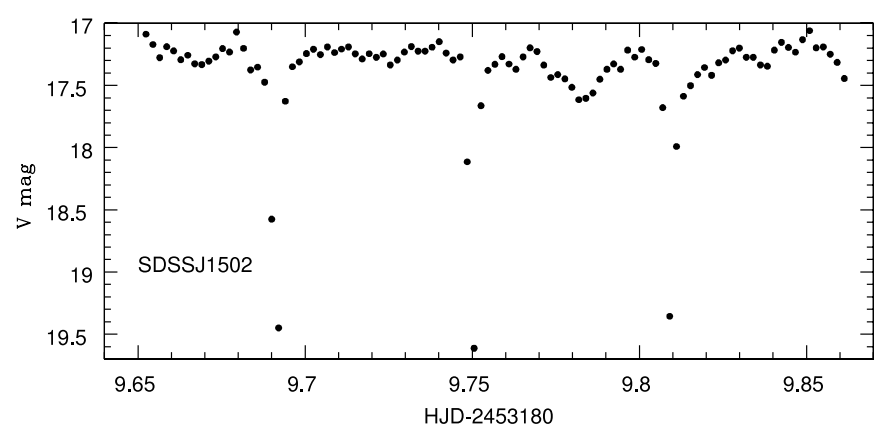

FIG. 2.-Light curve of SDSSJ 1502 showing three eclipses. Error bars are $\leq 0.02 \mathrm{mag}$, except during eclipses, when they are up to $0.09 \mathrm{mag}$.

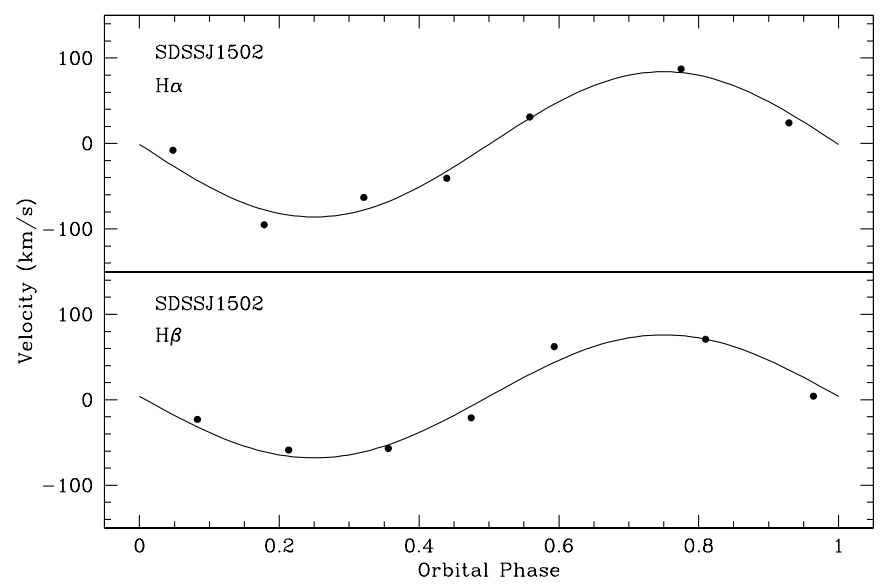

FIG. 3.-H $\alpha$ velocity curve of SDSSJ 1502 with the best-fit sinusoid (Table 4) superposed. 
TABLE 4

Radial Velocity Solutions

\begin{tabular}{|c|c|c|c|c|c|c|}
\hline SDSSJ & Line & $\begin{array}{c}P \\
\text { (minutes) }^{\mathrm{a}}\end{array}$ & $\gamma$ & $\begin{array}{c}K \\
\left(\mathrm{~km} \mathrm{~s}^{-1}\right)\end{array}$ & $T_{0}(\mathrm{JD} 2,450,000+)$ & $\sigma$ \\
\hline 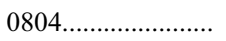 & $\mathrm{H} \alpha$ & $85 \pm 3$ & $39 \pm 1$ & $37 \pm 5$ & 3504.643 & 13 \\
\hline 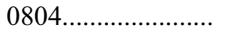 & $\mathrm{H} \beta$ & 85 & $14 \pm 3$ & $93 \pm 24$ & 3504.651 & 61 \\
\hline $1502 \ldots \ldots \ldots \ldots \ldots \ldots$ & $\mathrm{H} \alpha$ & 84.2 & $-1 \pm 1$ & $85 \pm 8$ & 3549.751 & 12 \\
\hline $1502 \ldots \ldots \ldots \ldots \ldots \ldots \ldots$ & $\mathrm{H} \beta$ & 84.2 & $4 \pm 1$ & $72 \pm 7$ & 3549.749 & 11 \\
\hline
\end{tabular}

a Period without uncertainty was fixed at that value during the fit.

image in the public archive for SDSSJ 0824 is not the image from which the psf mags were obtained). The differences are most noticeable in SDSSJ 0746, where there is a 3 mag difference between the psf $g$ mag of 18.2 and the spectrum $(g \sim 21.4)$; in SDSSJ 0824, with a 4 mag difference between photometry of 18.6 and the spectrum at 22.4 ; and in SDSSJ 2104, with a $3.5 \mathrm{mag}$ difference between the photometry of $17.1 \mathrm{mag}$ and the spectrum at $\sim 20.6 \mathrm{mag}$. SDSSJ 0746 is also fairly bright in the DSS, so this object could be a dwarf nova with frequent outbursts, while SDSSJ 0824 and 2104 are faint on the DSS. SDSSJ 0743 shows smaller differences ( $g$ of about 21.3 from its spectrum vs. 20.1 from its photometry), and the DSS image shows it to be very faint. SDSSJ 1653 (details below) was found to be brighter during its follow-up observations. Further long-term monitoring will be required to determine the outburst timescales for these systems and the actual amplitude of variation, as the available data could have been obtained during transitions between outburst and quiescence.

SDSSJ 1653.-Follow-up photometric observations of SDSSJ 1653 happened to occur at a time when it was about $3 \mathrm{mag}$ brighter than the SDSS photometry and spectroscopy. The light curve (Fig. 6) showed a periodic hump feature superposed on a declining trend that is best explained by superhumps (periodic modulations present in outbursting short-period dwarf novae with periods about $4 \%$ longer than the orbital period; Warner $1995)$ during the decline from an outburst. The observed superhump period of $1.58 \mathrm{hr}$ implies an orbital period near 91 minutes.

\subsection{Nova-like Stars with Strong He II}

The previously known Polars AR UMa (SDSSJ 1115) and AN $\mathrm{UMa}$ (SDSSJ 1104), as well as the previously known IP PQ Gem (SDSSJ 0751), all show strong He II, as expected for magnetic systems. The newly discovered low accretion rate Polar (SDSSJ 2048; Schmidt et al. 2005) does not show this line, as there is no hard X-ray production in the systems in which the accretion rate is so low that it is only via a wind (Szkody et al. 2004b). As

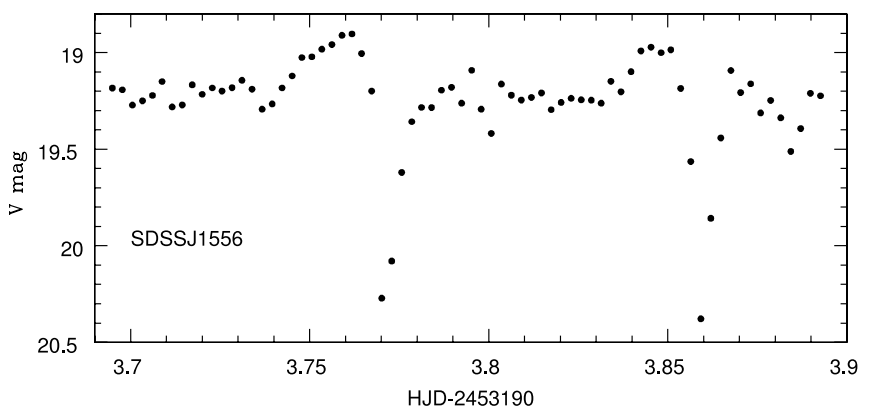

FIG. 4.- Light curve of SDSSJ 1556 showing two eclipses. Error bars outside of eclipse are $0.03-0.09 \mathrm{mag}$, and up to $0.22 \mathrm{mag}$ in eclipse. discussed in $\S 2$, UMa 6 (SDSSJ 0932) shows an abnormally large strength of $\mathrm{He}$ II, but there is no detailed study of this system available in the literature. Two systems, SDSSJ 1607 and 1659 , show continua and lines that are typical of high accretion rate SW Sex stars. As noted in $\S 3.2$, SDSSJ 1607 is eclipsing, and the eclipse shape is also consistent with those seen in SW Sex stars. SDSSJ 0754 and 0846 were candidates for Polars based on their line strengths and shapes. However, circular polarimetric observations (Table 2) reveal no polarization in either case. Thus, these objects could be IPs, and photometric monitoring is needed to determine if a spin period can be detected. Finally, SDSSJ 0759 shows a strength of He II that is larger than most dwarf novae but not quite up to the values typical for Polars or IPs, so the classification of this object is not clear.

\subsection{Systems Showing the Underlying Stars}

At low accretion rates, the contribution from an accretion disk can be minimal, and the underlying stars in the binary can be viewed. Figure 1 shows the broad absorption lines from a white dwarf in the systems SDSSJ 0745, 0804, 1035, 1202, 1227, and 1628. These systems are ideal candidates for short-period systems containing pulsating white dwarfs, as recently determined for four SDSS systems showing similar spectra (Warner \& Woudt 2004; Woudt \& Warner 2004; Gänsicke et al. 2006).
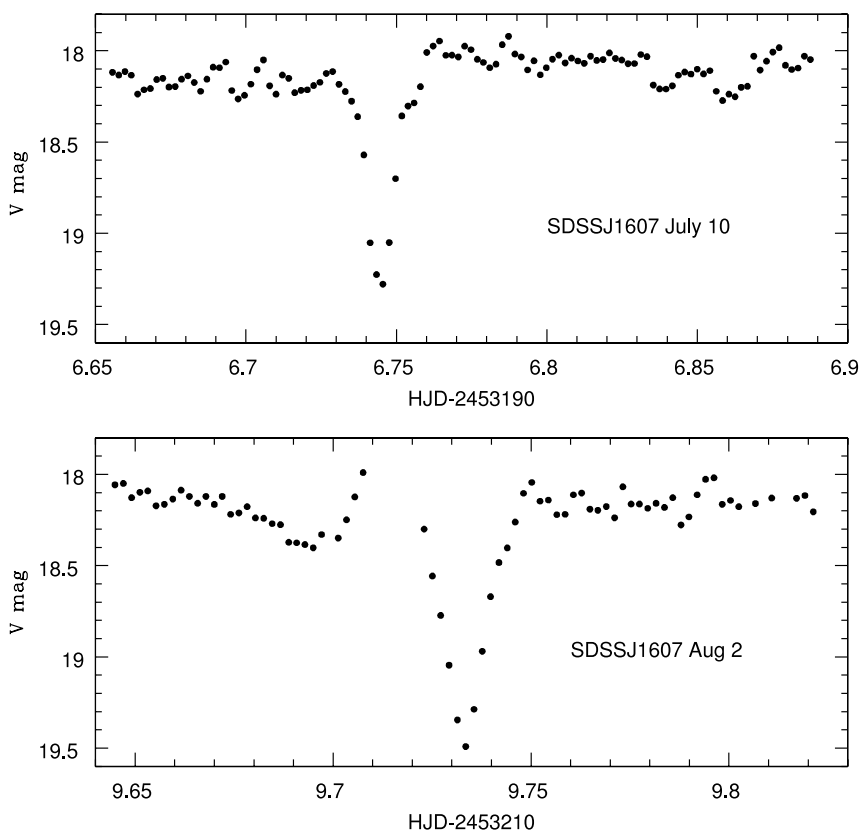

FIG. 5.- Light curve of SDSSJ 1607 showing the eclipses on each of two nights of observation. In July, error bars are 0.01 mag outside eclipse and $0.03 \mathrm{mag}$ in eclipse, while clouds resulted in larger errors for the August data, with errors up to 0.1 mag during and after the eclipse. 


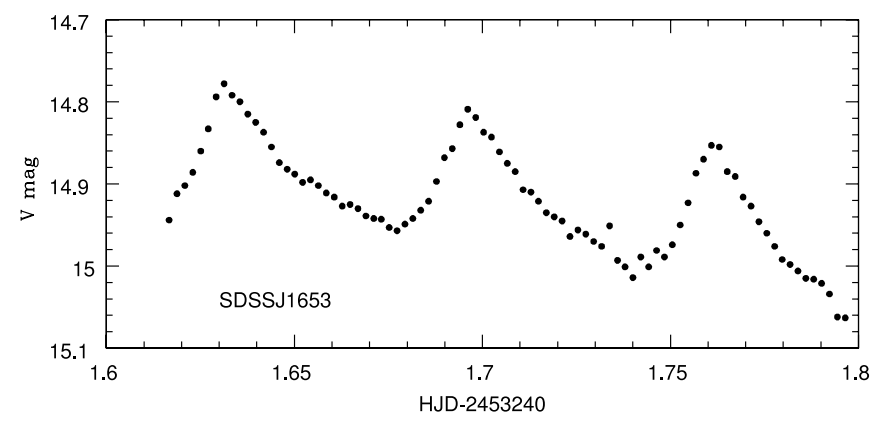

FIG. 6. - Light curve of SDSSJ 1653 showing three superhumps and a gradual overall decline in light from a likely outburst. Error bars on each point are $\leq 0.02$ mag.

These four comprise half of the known sample of eight ZZ Ceti stars contained in accreting close binaries. As the numbers grow, so does the ability to explore the interiors of accreting white dwarfs via asteroseismology and how the accretion affects the location of the instability strip.

Our follow-up observations of SDSSJ 0804 (described below) do not reveal pulsations but do show intriguing periodic variability. Figure 1 also indicates two likely long period systems (SDSSJ 2025 and 2335) based on the turnover in the blue spectral distribution, combined with the presence of narrow Balmer emission and $\mathrm{MgH}$ absorption.

SDSSJ 0804.-While our expectation was that we might see pulsations in the photometry of this object, the light curve (Fig. 7) instead shows highly unusual behavior. There is a 42.5 minute periodic oscillation in the data, as determined from the PDM routine in IRAF. This period is visible in Figure 7 with a 0.05 peak-to-peak amplitude for three cycles, followed by a rapid rise in brightness of the system by $0.5 \mathrm{mag}$, at which time the amplitude of the 42.5 minute variation increases to about $0.2 \mathrm{mag}$. The $3 \mathrm{hr}$ of time-resolved spectra (Table 2) did not show any noticeable changes in brightness throughout the series, although the flux levels of the APO spectra are comparable to the SDSS photometric value of 17.9 , while the SDSS spectra have a flux that is comparable to a magnitude near 18.3. This may indicate that the large 0.5 mag changes are persistent features of this system.

Since the emission lines have deep doubling, and the higher order Balmer lines are surrounded by increasing broad absorption, the double-Gaussian method was used to create a radial velocity curve. The best-fit sinusoid solution was obtained for a separation of the Gaussians of $1800 \mathrm{~km} \mathrm{~s}^{-1}$. Due to better $\mathrm{S} / \mathrm{N}$ data, the $\mathrm{H} \alpha$ line provided the best fit and determined a period of $85 \pm 3$ minutes. The period for $\mathrm{H} \beta$ was then fixed at this value and the best-fit sine solution determined (Table 4 ). Figure 8 shows

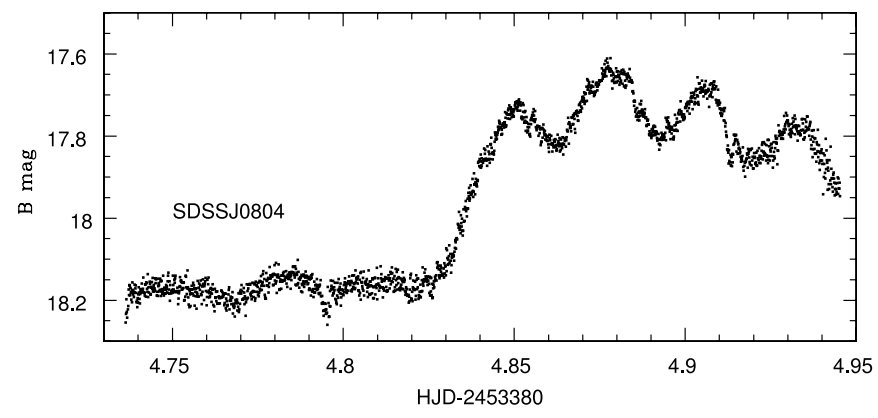

FIG. 7.- Light curve of SDSSJ 0804. Note the 42.5 minute oscillation, which increases in amplitude when the system undergoes a brightening of $0.5 \mathrm{mag}$. Error bars are $0.01 \mathrm{mag}$.

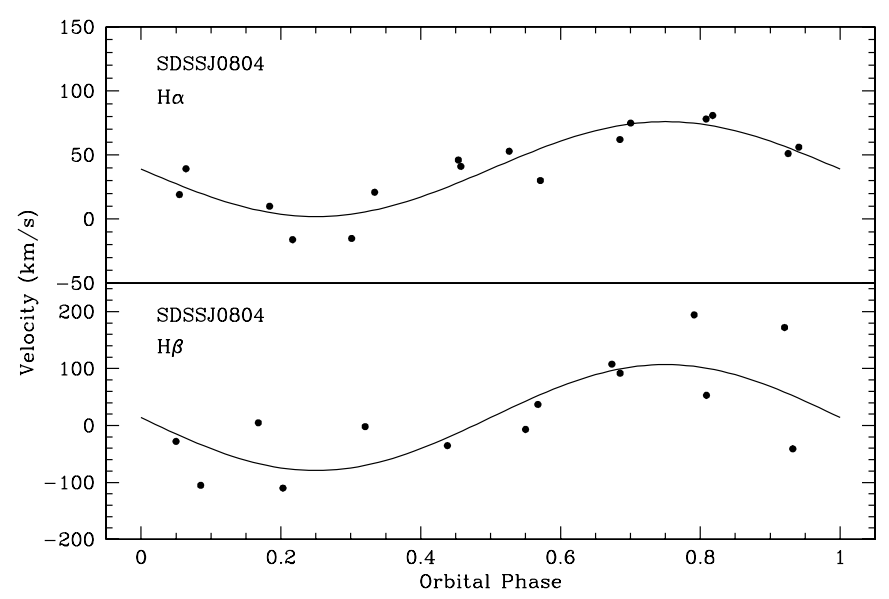

FIG. 8. - Velocity curves of SDSSJ 0804 with the best-fit sinusoids (Table 4).

the radial velocity curves for $\mathrm{H} \alpha$ and $\mathrm{H} \beta$. It is notable that the orbital period determined from the radial velocities is exactly twice the period that is evident in the photometry. Several systems with low mass transfer rates are known to have a double-humped modulation in their light curves (e.g., WZ Sge: Krzeminski \& Smak 1971; WX Cet: Rogoziacki \& Schwarzenberg-Czerny 2001) that is likely due to structure in the disk, possibly related to the hot spot and overflow of the stream to the opposite side of the disk. However, the large increase in brightness together with the increase in amplitude of the modulation has so far been reported in only one other system (SDSS J123813.73-033933.0; Zharikov et al. 2005), as far as we know. SDSSJ 1238 has a 40.25 minute variation with an amplitude of $0.15 \mathrm{mag}$, which is half the orbital period of 80.53 minutes. It shows a brightening of 0.4 mag on timescales of 7-12 hr, and the amplitude of the 40 minute variation increases during the bright state. The spectra of SDSSJ 0808 and 1238 are very similar in appearance as well.

The cause of this brightening could be mass transfer variations (although the source of the quasi-periodic nature is not clear), or it could be some sort of precession of the disk, as occurs in X-ray binaries (although this might be harder to explain, as the clock is variable). There are several short-period systems with low mass transfer rate that show long-term (hours) periodicities in their light curves. A summary of these is given in Table 5. They include systems containing pulsating white dwarfs, as well as those with normal white dwarfs. Obviously, there is some understanding of quiescent disk structure that is still missing.

\subsection{Other Disk Systems}

Based on the presence of $\mathrm{H} \alpha$ in emission (which is often doubled, indicating an accretion disk), combined with higher order Balmer lines in absorption that are too narrow to be due to

TABLE 5

Systems with Long Photometric Periods

\begin{tabular}{ccccl}
\hline \hline Object & $\begin{array}{c}P_{\text {orb }} \\
(\mathrm{hr})\end{array}$ & $\begin{array}{c}P_{\text {phot }} \\
(\mathrm{hr})\end{array}$ & $\begin{array}{c}\text { Amplitude } \\
(\mathrm{mag})\end{array}$ & \multicolumn{1}{c}{ Reference } \\
\hline GW Lib ............... & 1.28 & 2.08 & 0.05 & Woudt \& Warner (2002) \\
FS Aur............. & 1.43 & 3.4 & 0.24 & Tovmassian et al. (2002) \\
SDSSJ 1339 ....... & 1.38 & 5.7 & 0.025 & Gänsicke et al. (2006) \\
SDSSJ 1238........ & 1.34 & $7-12$ & 0.4 & Zharikov et al. (2005) \\
SDSSJ 0804 ....... & 1.5 & $>5$ & 0.4 & This paper \\
\hline
\end{tabular}




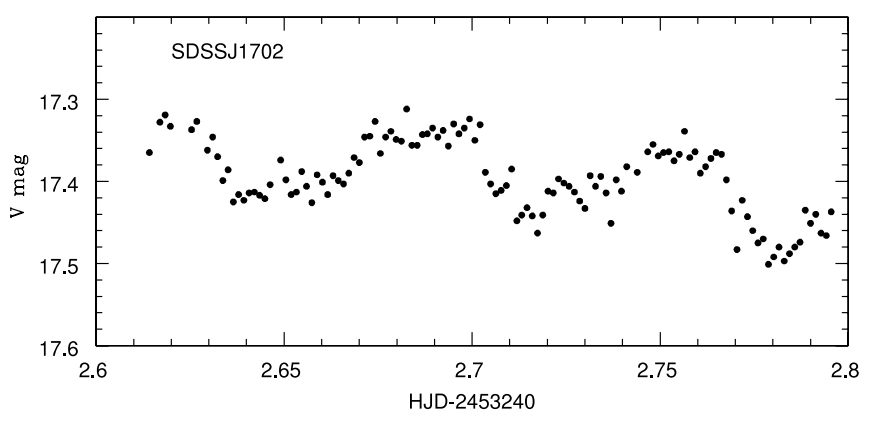

FIG. 9.- - Light curve of SDSSJ 1702 showing the sinusoidal variation at a period of 0.07 days. Error bars on each point are $\leq 0.02$ mag.

a white dwarf, the following systems are likely to be high accretion rate nova-like systems with periods longer than $3 \mathrm{hr}$ : SDSSJ 0026, 0840+04, 1219, and 1532. SDSSJ 1702 has broader lines with weak emission cores in $\mathrm{H} \beta$ and the higher Balmer series. The strong blue continuum and the weak lines would also indicate a high mass accretion rate. Follow-up photometry (Fig. 9) reveals a sinusoidal variation with a period of $1.6 \mathrm{hr}$ and a full amplitude of $0.1 \mathrm{mag}$. Further data will be required to determine if this variation is due to a hot spot in a system with a $1.6 \mathrm{hr}$ period, a double-humped variation in a system with a $3.2 \mathrm{hr}$ period, or a superhump-type modulation in a system like SDSSJ $210014.12+004446.0$ (Tramposch et al. 2005). This is somewhat of a puzzle, as the shape is not characteristic of a superhump (see Fig. 6), and the system was not noticeably brighter than the SDSS photometry or spectroscopy, which would rule out normal superhumps. On the other hand, high accretion rate systems do not usually have strong hot spots or double-peaked variations. The correct orbital period from a radial velocity curve should help to determine the correct interpretation.

\subsection{ROSAT Correlations}

There are eight systems with X-ray detections $(>2.5 \sigma)$ within the positional errors of the ROSAT All Sky Survey (RASS; Voges et al. 1999, 2000). Table 6 gives the X-ray count rates and exposure times for these sources. In addition, we also list the known Polar SDSSJ 2048 and IP SDSSJ 0758 (DW Cnc) with slightly lower detections (1.9 and $2.3 \sigma$, respectively), as they are very likely to be the RASS counterparts. Other than the known systems containing magnetic white dwarfs, which have the highest ROSAT count rates, the remaining low ROSAT count rates are typical for low accretion rate disk systems. The only object that is somewhat peculiar in Table 6 is SDSSJ 1138. Its spectrum shows very narrow lines and a Balmer decrement near recombination values, unlike the flat decrement indicative of optically thick lines that is apparent in most CVs. The ROSAT image shows two sources separated by about $3^{\prime}$ that are not resolved by the detection software. Thus, this X-ray detection should be used with caution.

\section{CONCLUSIONS}

With the 34 new CVs in this paper, the total number of new CVs found in SDSS is now 154, and the total number of CVs with SDSS spectra stands at 185 . This sample is now becoming large enough to provide a uniform database of spectra with which to compare characteristics across a wide variety of $\mathrm{CV}$ types. The increasing numbers of systems with very low accretion rates are providing new insights into how accretion oc-
TABLE 6

ROSAT DETECTIONS

\begin{tabular}{|c|c|c|c|c|}
\hline SDSSJ & $\begin{array}{c}\operatorname{ROSAT} \\
\left(\text { counts s }{ }^{-1}\right)^{\mathrm{a}}\end{array}$ & $\begin{array}{c}\text { Exposure } \\
\text { (s) }\end{array}$ & RXS & Type \\
\hline $0751 \ldots .$. & $2.96 \pm 0.27$ & 45 & $\mathrm{~J} 075117.6+144434=\mathrm{PQ} \mathrm{Gem}$ & IP \\
\hline $0758 \ldots .$. & $0.32 \pm 0.14$ & 21 & $\mathrm{~J} 075855.4+161801=\mathrm{DW} \mathrm{Cnc}$ & Pola \\
\hline $0836 \ldots .$. & $0.05 \pm 0.02$ & 206 & $\mathrm{~J} 083620.2+212109=\mathrm{CC} \mathrm{Cnc}$ & $\mathrm{DN}$ \\
\hline $0840 \ldots .$. & $0.08 \pm 0.03$ & 201 & J084024.8+220438 & \\
\hline $1100 \ldots$. & $0.03 \pm 0.01$ & 301 & $\mathrm{~J} 110014.7+131608$ & \\
\hline $1104 \ldots . .$. & $1.77 \pm 0.07$ & 405 & $\mathrm{~J} 110425.6+450319=\mathrm{AN} \mathrm{UMa}$ & Pola \\
\hline $1131 \ldots .$. & $0.05 \pm 0.01$ & 362 & $\mathrm{~J} 113121.8+432242$ & DN \\
\hline $1138 \ldots .$. & $0.03 \pm 0.01$ & 429 & $\mathrm{~J} 113826.5+061910$ & \\
\hline $1656 \ldots .$. & $0.04 \pm 0.01$ & 629 & $\mathrm{~J} 165658.7+212141$ & \\
\hline $2048 \ldots \ldots$ & $0.01 \pm 0.01$ & 458 & $\mathrm{~J} 204828.2+005022$ & Pola \\
\hline
\end{tabular}

${ }^{\text {a }}$ For a $2 \mathrm{keV}$ bremsstrahlung spectrum, 1 count $\mathrm{s}^{-1}$ corresponds to a $0.1-$ $2.4 \mathrm{keV}$ flux of about $7 \times 10^{-12} \mathrm{ergs} \mathrm{cm}^{-2} \mathrm{~s}^{-1}$.

curs in this regime. We have found that systems like the low accretion rate Polars can have accretion only via a wind. We now know of eight low accretion rate CVs that contain pulsating white dwarfs. There is obviously some disk structure that is causing periodic variation in mass transfer on 3-12 hr timescales in short orbital period systems. More surprises are likely in store from follow-up data on the faintest systems, but large apertures are needed for this work.

Several objects in this paper should be singled out for follow-up work with medium aperture (1-3 m) telescopes. Additional timeresolved spectra of SDSSJ 1131 are needed to determine if the cause of the strange $\mathrm{He}$ II $/ \mathrm{H} \beta$ ratio is a function of its $10 \mathrm{hr}$ orbital variability. More spectra are also desirable for SDSSJ 1607 (a likely SW Sex system with eclipses) and SDSSJ 1702 (a nova-like star with a prominent photometric variation) to determine the correct orbital periods.

Long stretches of photometry on SDSSJ 0804 can determine the cause of the unusual modulations in its light curve and whether the large-scale increases in brightness have a periodic timescale on the order of hours, as evident in SDSSJ 1238, as well as other short period systems. For those with fast-readout CCDs, photometry of the objects showing the white dwarf can determine if they contain pulsating ZZ Ceti stars.

Funding for the creation and distribution of the SDSS Archive has been provided by the Alfred P. Sloan Foundation, the Participating Institutions, the National Aeronautics and Space Administration, the National Science Foundation, the US Department of Energy, the Japanese Monbukagakusho, and the Max Planck Society. The SDSS Web site is at http://www.sdss.org. The SDSS is managed by the Astrophysical Research Consortium for the Participating Institutions. The Participating Institutions are the University of Chicago, Fermilab, the Institute for Advanced Study, the Japan Participation Group, The Johns Hopkins University, the Korean Scientist Group, Los Alamos National Laboratory, the Max Planck Institute for Astronomy, the Max Planck Institute for Astrophysics, New Mexico State University, the University of Pittsburgh, Princeton University, the US Naval Observatory, and the University of Washington. P. S. and N. M. S. acknowledge support from NSF grant AST 02-05875. Studies of magnetic stars and stellar systems at Steward Observatory are supported by the NSF through AST 97-30792. 
Abazajian, K., et al. 2003, AJ, 126, 2081 2004, AJ, 128,502 2005, AJ, 129, 1755

Adelman-McCarthy, J. K., et al. 2006, ApJS, in press

Fukugita, M., Ichikawa, T., Gunn, J. E., Doi, M., Shimasaku, K., \& Schneider, D. P. 1996, AJ, 111, 1748

Gänsicke, B. T., et al. 2006, MNRAS, in press

Gunn, J. E., et al. 1998, AJ, 116, 3040 2005, AJ, submitted

Hogg, D. W., Finkbeiner, D. P., Schlegel, D. J., \& Gunn, J. E. 2001, AJ, 122, 2129

Howell, S. B., Nelson, L. A., \& Rappaport, S. 2001, ApJ, 550, 897

Ivezić, Ž., et al. 2004, Astron. Nachr., 325, 583

Krzeminski, W., \& Smak, J. 1971, Acta Astron., 21, 133

Lupton, R. 2005, AJ, submitted

Lupton, R. H., Gunn, J. E., Ivezić, Ž., Knapp, G. R., Kent, S. M., \& Yasuda, N.

2001, in ASP Conf. Ser. 238, Astronomical Data Analysis Software and Systems

X, ed. F. R. Harnden, Jr., F. A. Primini, \& H. E. Payne (San Francisco: ASP), 269

Lupton, R. H., Gunn, J. E., \& Szalay, A. 1999, AJ, 118, 1406

Munari, U., Zwitter, T., \& Bragaglia, A. 1997, A\&AS, 122, 495

Nather, R. E., \& Mukadam, A. S. 2004, ApJ, 605, 846

Pier, J. R., Munn, J. A., Hindsley, R. B., Hennessy, G. S., Kent, S. M., Lupton,

R. H., \& Ivezić, Ž. 2003, AJ, 125, 1559

Rogoziacki, P., \& Schwarzenberg-Czerny, A. 2001, MNRAS, 323, 850

Schmidt, G. D., et al. 2005, ApJ, 630, 1037

Shafter, A. W. 1983, ApJ, 267, 222

\section{REFERENCES}

Smith, J. A., et al. 2002, AJ, 123, 485

Stoughton, C., et al. 2002, AJ, 123, 485

Szkody, P., et al. 2002, AJ, 123, 430

2003a, ApJ, 583, 902

2003b, AJ, 126, 1499

$2004 \mathrm{a}, \mathrm{AJ}, 128,1882$

$2004 \mathrm{~b}, \mathrm{AJ}, 128,2443$

2005, AJ, 129, 2386

Tovmassian, G., Zharikov, S., Neustroev, V. V., Michel, R., \& Greiner, J. 2002, in ASP Conf. Ser. 261, The Physics of Cataclysmic Variables and Related Objects, ed. B. T. Gänsicke, K. Beuermann, \& K. Reinsch (San Francisco: ASP), 557

Tramposch, J., et al. 2005, PASP, 117, 262

Tucker, D., et al. 2005, AJ, submitted

Voges, W., et al. 1999, A\&A, 349, 389 2000, IAU Circ. 7432

Warner, B. 1995, Cataclysmic Variable Stars (Cambridge: Cambridge Univ. Press) Warner, B., \& Woudt, P. 2004, in ASP Conf. Ser. 310, Variable Stars in the Local Group, ed. D. W. Kurtz \& K. R. Pollard (San Francisco: ASP), 382 Wickramasinghe, D. T., \& Ferrario, L. 2000, PASP, 112, 873

Woudt, P., \& Warner, B. 2002, Ap\&SS, 282, 433 2004, MNRAS, 348, 599

York, D. G., et al. 2000, AJ, 120, 1579

Zharikov, S. V., Tovmassian, G. H., Neustroev, V., Michel, R., \& Napiwotzki, R. 2005, in ASP Conf. Ser. 330, The Astrophysics of Cataclysmic Variables and Related Objects, ed. J.-M. Hameury \& J.-P. Lasota (San Francisco: ASP), 327 